\title{
Rabbit Model of Extending Knee Joint Contracture: Progression of Joint Motion Restriction and Subsequent Joint Capsule Changes after Immobilization
}

\author{
Yun Zhou, MD, PhD ${ }^{1,2}$ Quan Bing Zhang, MM ${ }^{1}$ Hua Zhang Zhong, MM ${ }^{2}$ YiLiu, MD ${ }^{1}$ Jun Li, MD, PhD ${ }^{2}$ \\ Hao Lv, MM² Jue Hua Jing, MD, $\mathrm{PhD}^{2}$
}

1 Department of Rehabilitation Medicine, The Second Hospital of Anhui Medical University, Hefei, People's Republic of China

2 Department of Orthopedics, The Second Hospital of Anhui Medical University, Hefei, People's Republic of China

Address for correspondence Yun Zhou, MD, PhD, Department of Rehabilitation Medicine, The Second Hospital of Anhui Medical University, Hefei 230601, People's Republic of China (e-mail: zhouyunanhui@sina.com).

J Knee Surg 2020;33:15-21.

\begin{abstract}
Keywords

- joint contracture

- animal model

- joint range of motion

- joint capsule

- rabbit

This study aimed to develop a rabbit model of knee contracture in extension and investigate the natural history of motion loss and time-dependent changes in the joint capsule after immobilization. We immobilized the unilateral knee joints of 32 rabbits by maintaining the knee joint in a plaster cast at full extension. Eight rabbits were euthanized at 2, 4, 6, and 8 weeks after casting, respectively, and the lower extremities were disarticulated at the hip joint. Eight control group rabbits that did not undergo immobilization were also examined. We assessed the progression of joint contracture by measuring the joint range of motion, evaluating the histologic alteration of the capsule, and assessing the mRNA levels of transforming growth factor $\beta 1$ (TGF- $\beta 1$ ) in the anterior and posterior joint capsules. After 2 weeks of joint immobilization, the knee joint range of motion was limited, the synovial membrane of the suprapatellar and posterior joint capsules was thickened, the collagen deposition was increased, and the mRNA levels of TGF- $\beta 1$ were elevated in the anterior and posterior joint capsules. These changes progressed rapidly until 6 weeks of immobilization and may advance slowly after 6 weeks. Joint contracture developed at the early stage of immobilization and progressed over time. The changes in the anterior and posterior joint capsules after joint immobilization may contribute to the limitation in flexion. The elevated mRNA expression of TGF- $\beta 1$ may be related to joint capsule fibrosis and may be one of the causes of joint contracture.
\end{abstract}

Joint contracture is defined as a decrease in joint range of motion (ROM) due to various reasons. ${ }^{1-3}$ The formation of joint contracture may limit the activities of daily living, and some patients eventually develop lifelong disabilities. Joint contracture is currently considered to be one of the most troublesome problems in orthopaedic trauma and rehabilitation medicine. Clinically, many diseases (including joint damage, joint immobilization, and some nervous system diseases) can result in the development of joint contracture, of which joint immobilization is considered to be the most common one. ${ }^{4,5}$ Joint immobilization is often used as an orthopaedic treatment for patients with joint injuries and other musculoskeletal disorders. $^{6,7}$ However, the long-term side effects of joint immobilization are incidental. After a joint was immobilized for a long time, connective tissue shortening, muscle atrophy, and weakening strength of muscles around the joint may be caused, inevitably leading to restricted joint activities and then influencing the patients' qualities of life..$^{8-11}$

The development of immobilization-induced joint contracture may be influenced by two anatomical components around the joint: arthrogenic components and myogenic components. The arthrogenic components are injuries to the bone, cartilage, received

April 28, 2018

accepted after revision

October 28, 2018

published online

December 18, 2018
Copyright $\odot 2020$ by Thieme Medical Publishers, Inc., 333 Seventh Avenue, New York, NY 10001, USA. Tel: +1(212) 584-4662.
DOI https://doi.org/ 10.1055/s-0038-1676502. ISSN 1538-8506. 
capsule, and synovial membrane, while the myogenic components are lesions of the muscle, tendon, and fascia. ${ }^{6,12}$ Arthrogenic components, particularly regarding the joint capsule, are reportedly important factors in the formation of immobilization-induced joint contracture. ${ }^{6,13,14}$ Reported changes in the capsule after immobilization include proliferation of connective tissues within the joint space, and adhesions in the capsule and synovial membrane. ${ }^{15,16}$ Some studies reported that joint capsule fibrosis may occur in the posttraumatic joint contracture caused by joint immobilization in flexion in rabbit or rat models. ${ }^{17,18}$ Furthermore, Hagiwara et al ${ }^{12}$ reported that the increased elasticity and structural changes of the posterior joint capsule may result in limited extension after a long period of immobilization in flexion. Most of studies involving joint contracture investigated the histological and molecular changes of joint contracture using the traumatic flexion joint contracture animal models, which involved two factors (including trauma and joint immobilization) that may influence the development of joint contracture. Nevertheless, some studies showed that patients with conditions limiting their mobility but without traumatic or inflammatory conditions are also at a high risk for joint contracture. $6,13,19$

During the process of joint contracture formation, the expressions of many growth factors, cytokines, and matrix metalloproteinases may be changed, which can contribute to joint contracture. ${ }^{20,21}$ Hildebrand et $\mathrm{al}^{22}$ reported elevated expressions of collagen types I and III and transforming growth factor $\beta 1$ (TGF- $\beta 1$ ) in the experimental posterior joint capsules compared with control capsules in a rabbit posttraumatic contracture model. Monument et $\mathrm{al}^{23}$ reported significant increase in the mRNA and protein levels of a-SMA, TGF- $\beta 1$, tryptase, and collagen types I and III in the operated contracture group compared with the control group. Among the cytokines that may play important roles in the formation of joint contracture, TGF- $\beta 1$ is considered to be the most crucial one affecting the development of fibrotic processes, as it modulates fibroblast function to promote matrix preservation. ${ }^{7}$

Extending nontraumatic immobilization-induced joint contracture is a challenging problem in clinical practice. However, few studies have investigated this type of joint contracture. The study of extending joint contracture is of great importance for the prevention and treatment of the disease. In the present study, we established a model of rabbit knee contracture in extension using plaster casting. Serial changes in the joint ROM, the thickening and fibrosis of the joint capsule, and the mRNA levels of TGF- $\beta 1$ in the joint capsule over time were then evaluated. We aimed to probe into the histological and molecular changes of anterior and posterior joint capsules evoked by immobilization, to gain a better understanding in regard to the underlying molecular cause of immobilization-induced joint contracture.

\section{Materials and Methods}

\section{Experimental Design}

The present study was approved by the Institutional Animal Care and Use Committee of Anhui Medical University. In total, the present study used 40 male New Zealand white

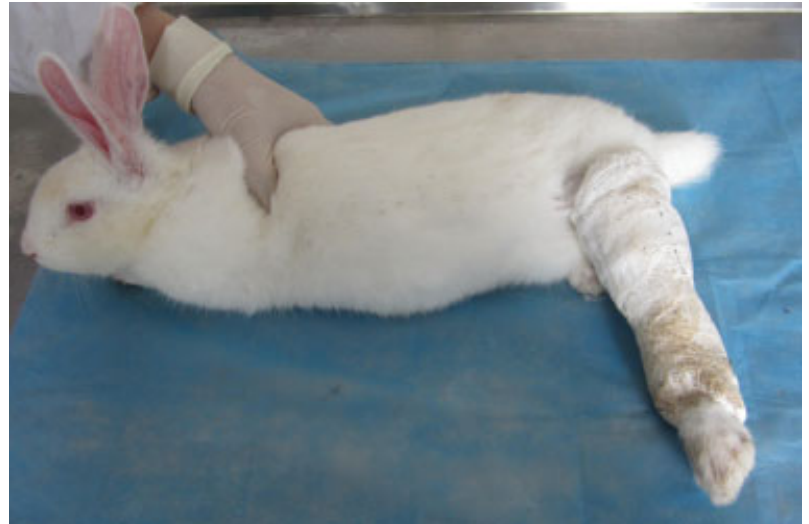

Fig. 1 Modeling of knee extension contracture. The rabbits underwent unilateral immobilization of the knee joint at full extension using a plaster cast from the groin to the proximal toes.

rabbits (obtained from the Laboratory Animal Center of Anhui Medical University, Hefei, China; age, 3-4 months; weight, $2-2.5 \mathrm{~kg}$ ). Thirty-two of the 40 rabbits were anesthetized by intravenous administration into the ear vein of 30 $\mathrm{mg} / \mathrm{kg}$ sodium pentobarbital. Joint interventions were performed on the left knee under general anesthesia. The 32 rabbits underwent unilateral immobilization of the left knee joint at full extension using a plaster cast from the groin to

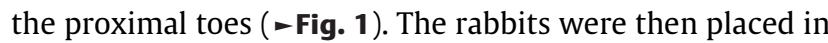
cages, with unrestricted activity and free access to water and food. The plaster casts were removed at 2, 4, 6, and 8 weeks after immobilization, with eight rabbits in each time cohort. A control group of eight rabbits that did not undergo immobilization were examined at the beginning of the experiment.

\section{Measurements of the Synovial Thickness of the Suprapatellar and Posterior Joint Capsule}

In view of the possible influences of synovial thickening on the limitations of joint ROM, color Doppler ultrasonic diagnostic instrument with the frequency of 10 to $22 \mathrm{MHz}$ was used for the measurements of the synovial thickness of suprapatellar and posterior joint capsules before the rabbits were euthanized.

\section{Range of Motion Measurements}

The rabbits were euthanized via a sodium pentobarbital overdose after the synovial thickness of the suprapatellar and posterior joint capsules was measured. A mechanical goniometer (arthrometer) was built and tested to measure the knee joint angle (-Fig. 2). The femur was secured using a metal clamp and a platform beside the disc. To avoid friction between the femur and the disc while the measurements were conducted, the platform and the middle part of the disc were designed to be a little higher than the periphery of the disc. Metal fixing clamps were used to fix the proximal and distal parts of the tibia on the disc. A precise digital force gauge was fixed on a pedestal which was placed on the slideway equipment. A string was used to connect the groove of the disc and the digital force gauge. All limbs started with 0 degree of extension before the force was applied. When the driving wheel 


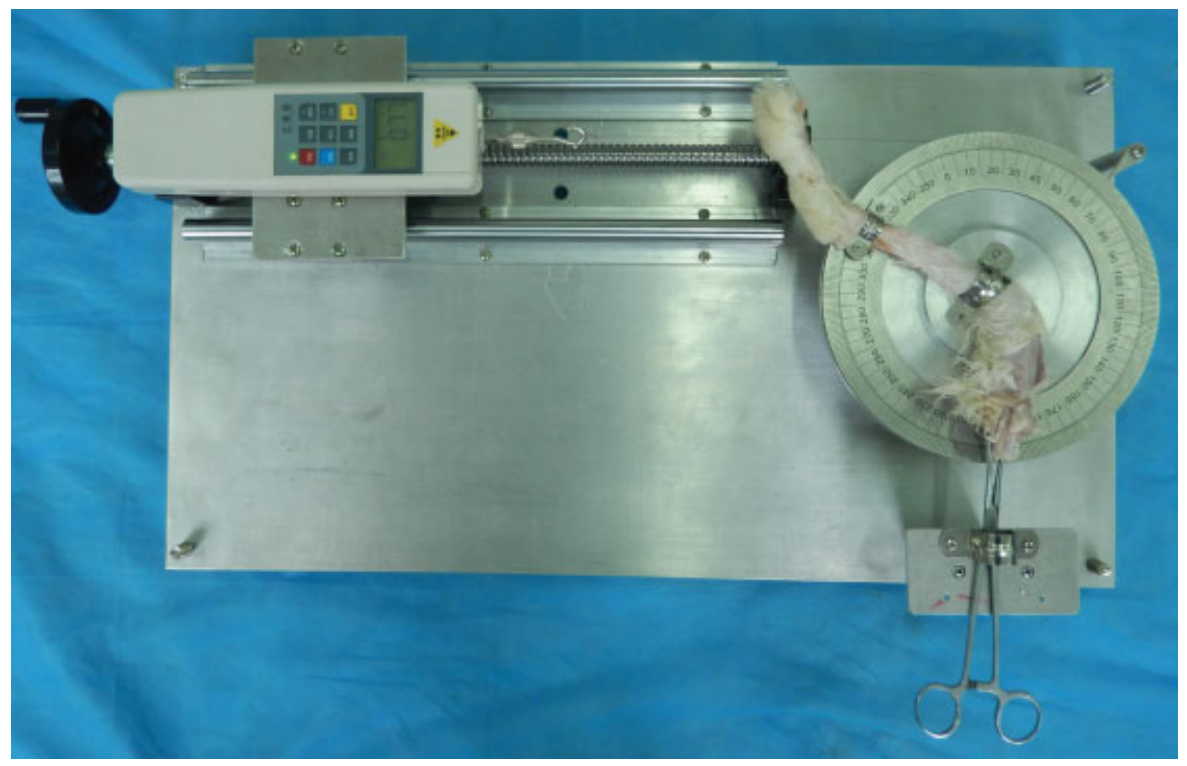

Fig. 2 The mechanical arthrometer used in the present study.

was turned, the disc was twisted, and the tibia was indirectly turned, while the femur remained static. The force can be read on the screen of the digital force gauge, and the angle variation between the femur and the tibia was calculated according to the scale on the disc as the measurements were performed. As the radius of the disc remained unchanged, the torque which was calculated via multiplying the force by the constant radius of the disc depended on the force applied. Before the former experiment, the ROM was measured in several hindlimbs of normal rabbits using the mechanical goniometer. We found that the knee joint can be pulled to $\sim 140$ degrees with a torque of $0.077 \mathrm{Nm}$, after that an increase in torque can result in very small angle increase. Consequently, in our formal experiments, $0.077 \mathrm{Nm}$ was used as a standardized torque value to measure the knee joint ROM. To ensure experimental accuracy, two examiners independently conducted the ROM measurements, and repeated these measurements three times for each limb. The examiners were blinded to each other's scores. Reported values were the mean of the six measurements taken by both examiners. Thus, a larger flexion angle actually represented a less severe contracture or loss of motion. The lack of flexion was referred to as an extension contracture.

\section{Tissue Preparation}

The anterior and posterior joint capsules were harvested from the knee and partitioned into two equal samples after the ROM measurements at each time point. Some samples used for Masson staining were fixed with $4 \%$ paraformaldehyde overnight at $4^{\circ} \mathrm{C}$, while other samples used for semiquantitative reverse transcription polymerase chain reaction (RT-PCR) were frozen in liquid nitrogen and then stored at $-70^{\circ} \mathrm{C}$.

\section{Masson Staining}

The fixed joint capsule specimens were dehydrated in graded alcohol and then embedded in paraffin. A series of 5.0- $\mu \mathrm{m}$ sections were cut, deparaffinized, and stained with the Masson method to assess the level of collagen deposition. The Masson- stained sections were then observed under $100 \times$ magnification, and six randomly selected fields were analyzed to determine the percentage of the blue area that indicated collagen deposition using Image ProPlus software (Media Cybernetics, Silver Spring, MD). The average percentage of the blue area from each slice was calculated and used as a measure of collagen deposition.

\section{RNA Extraction and Semiquantitative Reverse Transcription Polymerase Chain Reaction}

Total RNA was extracted from the joint capsule specimens using TRIzol reagent (Invitrogen, Carlsbad, CA) according to the manufacturer's instructions. The reverse transcription was performed using a RevertAid First Strand cDNA Synthesis Kit (Thermo Scientific, San Jose, CA) according to the manufacturer's instructions. Rabbit-specific primers (GAPDH: F-TCA CCA TCT TCC AGG AGC GA and R-CAC AAT GCC GAA GTG GTC GT; TGF- $\beta 1$ : F-CGG CAG CTG TAC ATT GAC TT and R-AGC GCA CGATCA TGT TGG AC) were used as described previously. ${ }^{23,24}$ PCR products were then separated by electrophoresis on $2.0 \%$ agarose gel. Images were captured using the Gel-Doc image analysis system (Bio-Rad, CA). To ensure experimental accuracy, all reactions were performed in triplicate. GAPDH was used as an internal control, and the gray ratio of TGF- $\beta 1 /$ GAPDH was used to reflect the relative expression levels. The results were expressed as the mean \pm standard deviation (SD).

\section{Statistical Analyses}

Data were presented as the mean $\pm \mathrm{SD}$. All data were analyzed using analysis of variance (ANOVA) with a post hoc Student-Newman-Keuls' (SNK) test for comparison between individual groups. The ROM, synovial thickness of the joint capsule, deposition of collagen, and mRNA expressions of TGF- $\beta 1$ were compared among all groups via ANOVA. An $\alpha$ less than 0.05 was chosen as the significance level for these statistical analyses. 


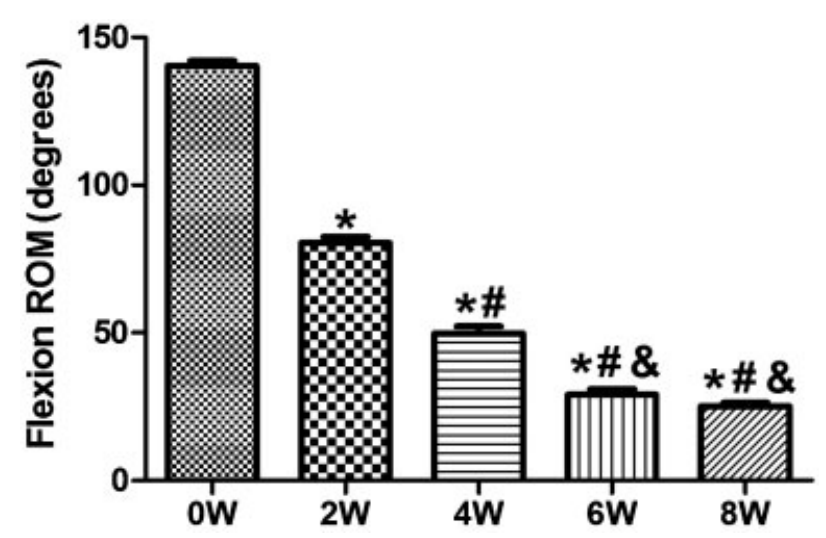

Fig. 3 Knee flexion range of motion (ROM) in each group. $0 \mathrm{~W}=$ control group that did not undergo immobilization; $2 \mathrm{~W}=$ rabbits that underwent 2 weeks of immobilization; $4 \mathrm{~W}=$ rabbits that underwent 4 weeks of immobilization; $6 \mathrm{~W}=$ rabbits that underwent 6 weeks of immobilization; $8 \mathrm{~W}=$ rabbits that underwent 8 weeks of immobilization. ${ }^{*} p<0.05$ versus the OW group; ${ }^{\#} p<0.05$ versus the $2 \mathrm{~W}$ group; ${ }^{\circledR} p<0.05$ versus the $4 \mathrm{~W}$ group.

\section{Results}

The knee flexion ROM of the five groups is illustrated in - Fig. 3 (0 week, $140.86 \pm 4.88$ degrees; 2 weeks, $80.43 \pm 5.96$ degrees; 4 weeks, $49.75 \pm 6.96$ degrees; 6 weeks, $29.05 \pm 5.24$ degrees; and 8 weeks, $25.21 \pm 3.21$ degrees). The ROM of the knee joints gradually decreased over time. After 2 weeks of immobilization, knee flexion ROM was decreased compared with the control group $(p<0.05)$. Furthermore, there was a significant difference between the ROM of rabbits immobilized for 4 weeks compared with those immobilized for 2 weeks ( $p<0.05)$. There was also a significant difference in ROM between rabbits immobilized for 6 versus 4 weeks $(p<0.05)$. However, there was no significant difference in the ROM of the knee joints of rabbits immobilized for 8 versus 6 weeks $(p>0.05)$.

According to the B ultrasonic examination (-Fig. 4), the synovial membrane of both the suprapatellar and posterior joint capsules gradually thickened after immobilization. Both the synovial thicknesses of the suprapatellar and posterior joint capsules were increased at 2 weeks after immobilization, and remained increased for the duration of the study. Regarding the effect of time, the experimental 2-week synovial thicknesses of the suprapatellar and posterior joint capsules were greater than the control 0 -week values ( $p<0.05$ ). The experimental 4 week thicknesses of the suprapatellar and posterior joint capsules were greater than the experimental 2-week values $(p<0.05)$. The experimental 6-week thicknesses of the suprapatellar and posterior joint capsules were greater than the control 4 -week values $(p<0.05)$. However, there was no significant difference in the thicknesses of both the suprapatellar and posterior joint capsules between the knee joints of rabbits immobilized for 8 versus 6 weeks $(p>0.05)$.

Masson staining showed that the collagen deposition in the anterior and posterior joint capsules gradually increased after immobilization (-Fig. 5). In both the anterior and posterior joint capsules, the collagen deposition in the synovial membrane was increased at 2 weeks after immobilization and remained elevated for 8 weeks. In regard to the impact of immobilization period, the experimental 2-week collagen deposition in the anterior and posterior joint capsules was greater than the control 0 -week value $(p<0.05)$, the experimental 4-week collagen deposition in the anterior and posterior joint capsules was greater than the experimental 2-week value $(p<0.05)$, and the experimental 6 -week collagen deposition in the anterior and posterior joint capsules was greater than the control 4 -week value $(p<0.05)$. However, there was no significant difference in the collagen deposition in both the anterior and posterior joint capsules between the knee joints of rabbits immobilized for 8 versus 6 weeks ( $p>0.05$ ).

-Fig. 6 illustrates the average mRNA levels of TGF- $\beta 1$ relative to the average band intensity of GAPDH in the five rabbit groups. A significant increase in mRNA levels was seen for TGF- $\beta 1$ in the joint capsule after immobilization. The mRNA levels of this growth factor in the experimental joint capsules were greater than those in the control group from 2 weeks after joint immobilization, and remained elevated until 8 weeks.
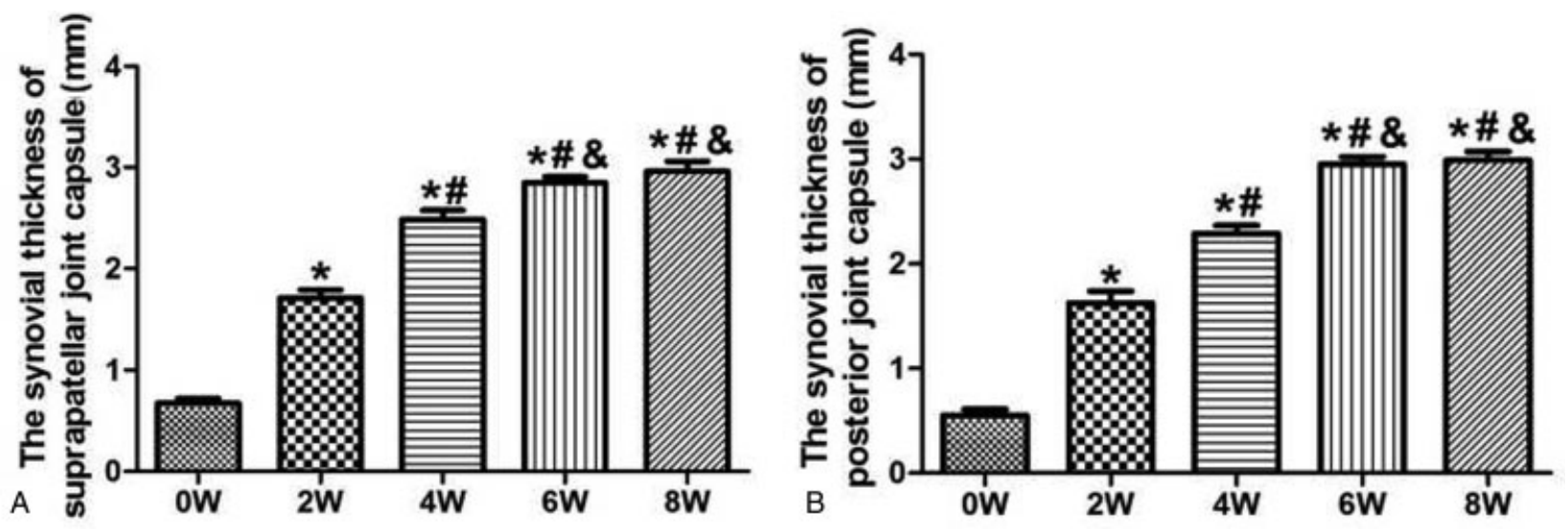

Fig. 4 (A, B) The synovial thicknesses of the suprapatellar and posterior joint capsules in each group. 0W = control group that did not undergo immobilization; $2 \mathrm{~W}=$ rabbits that underwent 2 weeks of immobilization; $4 \mathrm{~W}=$ rabbits that underwent 4 weeks of immobilization; $6 \mathrm{~W}=$ rabbits that underwent 6 weeks of immobilization; $8 \mathrm{~W}=$ rabbits that underwent 8 weeks of immobilization. ${ }^{*} p<0.05$ versus the $0 \mathrm{~W}$ group; ${ }^{\#} p<0.05$ versus the $2 \mathrm{~W}$ group; ${ }^{\&} p<0.05$ versus the $4 \mathrm{~W}$ group. 
A
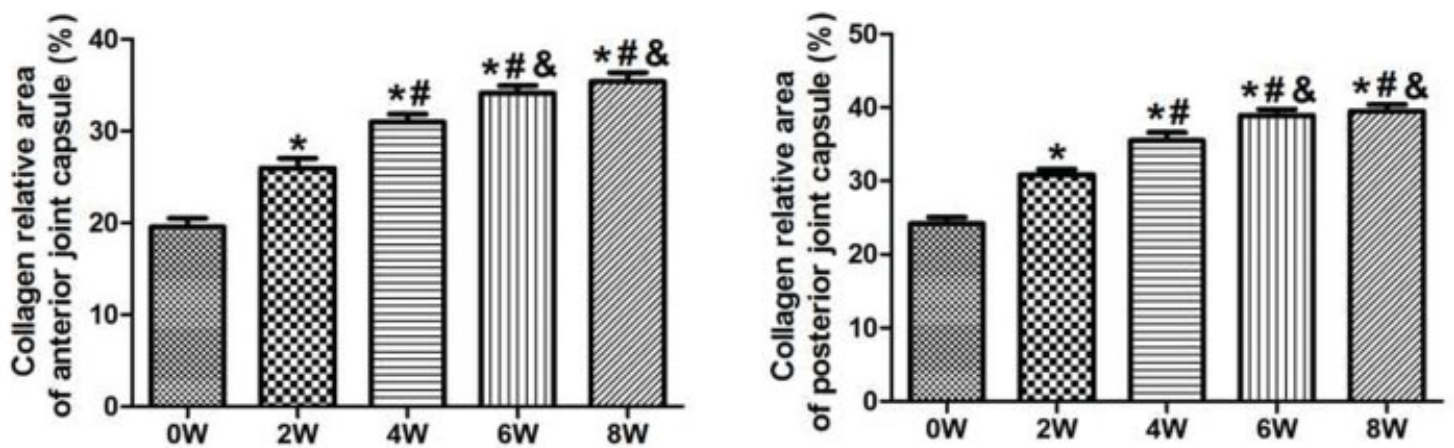

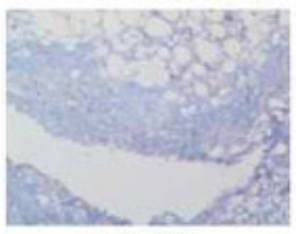

OW (a)

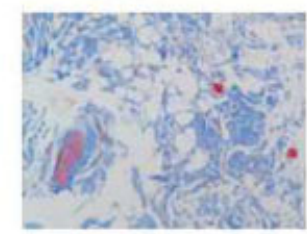

OW (p)

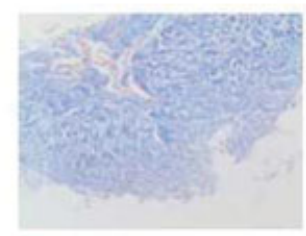

$2 \mathrm{~W}$ (a)

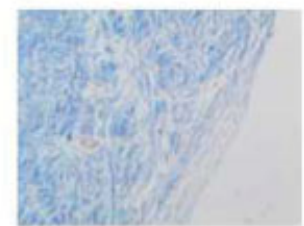

$2 W(p)$

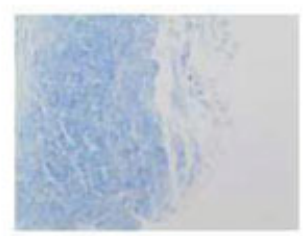

4W (a)

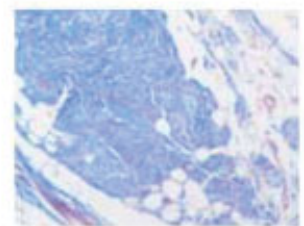

$4 W$ (p)

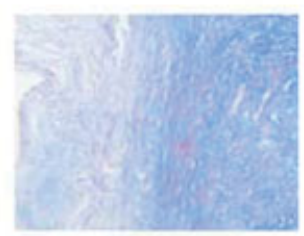

$6 \mathrm{~W}$ (a)

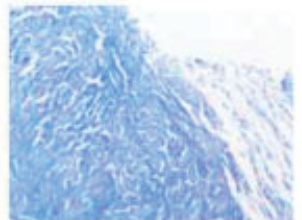

6W (p)

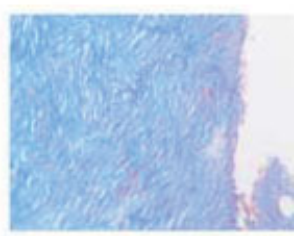

8W (a)

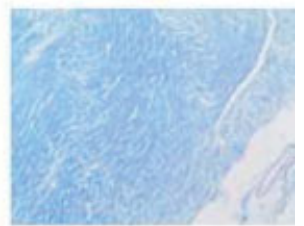

8W (p)

Fig. 5 (A, B) Collagen deposition area of the anterior and posterior joint capsules in each group. 0W = control group that did not undergo immobilization; $2 \mathrm{~W}=$ rabbits that underwent 2 weeks of immobilization; $4 \mathrm{~W}=$ rabbits that underwent 4 weeks of immobilization; $6 \mathrm{~W}=$ rabbits that underwent 6 weeks of immobilization; $8 \mathrm{~W}=$ rabbits that underwent 8 weeks of immobilization. a, anterior joint capsule; $\mathrm{p}$, posterior joint capsule. ${ }^{*} p<0.05$ versus the $0 \mathrm{~W}$ group; ${ }^{*} p<0.05$ versus the $2 \mathrm{~W}$ group; ${ }^{\&} p<0.05$ versus the $4 \mathrm{~W}$ group.
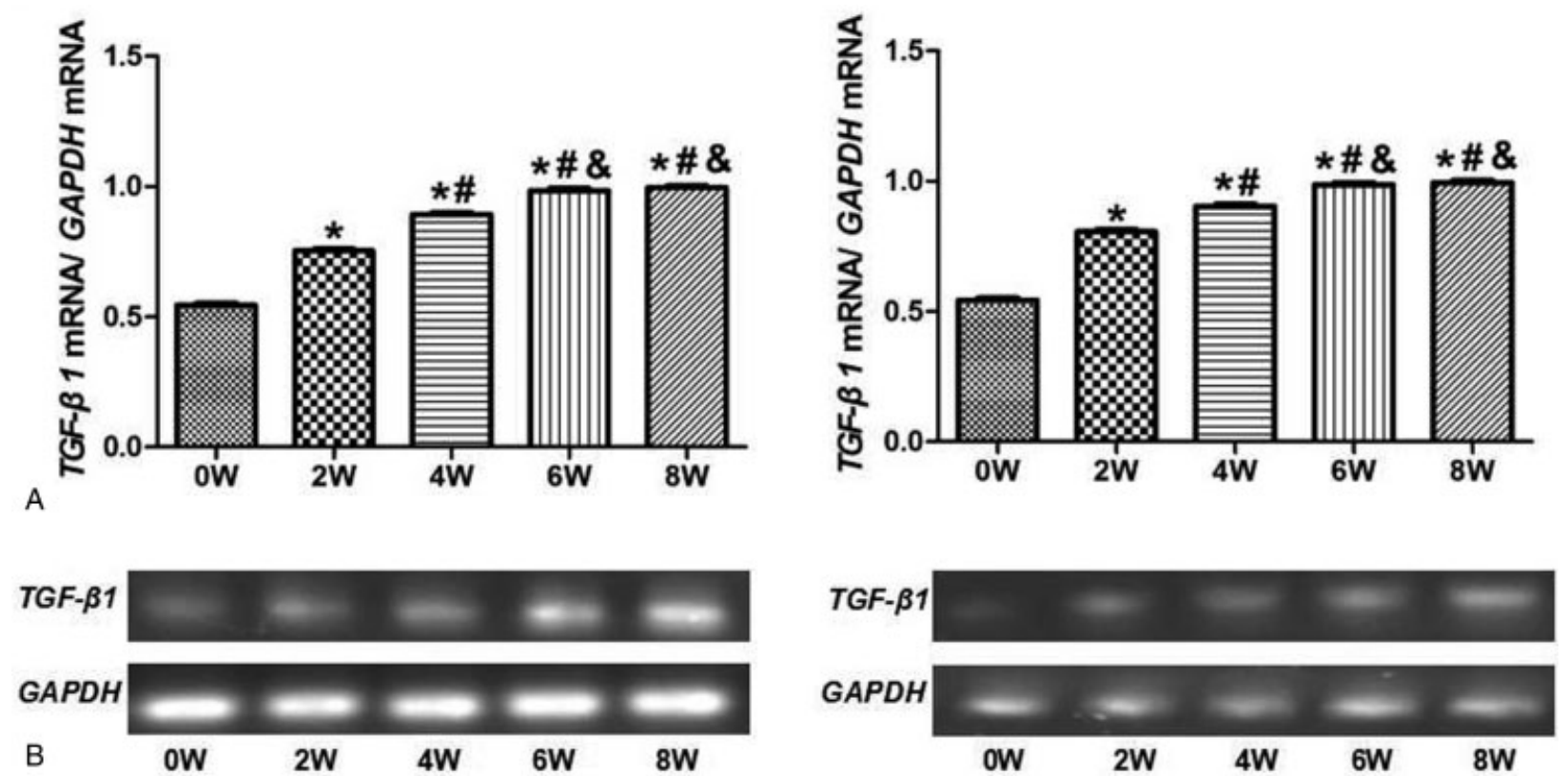

Fig. 6 Graphical representation of the transforming growth factor $\beta 1$ (TGF- $\beta 1$ ) mRNA levels relative to GAPDH in the anterior and posterior joint capsules (A), and a photograph of the TGF- $\beta 1$ and GAPDH mRNA band intensities (B). $0 \mathrm{~W}=$ control group that did not undergo immobilization; $2 \mathrm{~W}=$ rabbits that underwent 2 weeks of immobilization; $4 \mathrm{~W}=$ rabbits that underwent 4 weeks of immobilization; $6 \mathrm{~W}=$ rabbits that underwent 6 weeks of immobilization; $8 \mathrm{~W}=$ rabbits that underwent 8 weeks of immobilization. ${ }^{*} p<0.05$ versus the $0 \mathrm{~W}$ group; ${ }^{\#} p<0.05$ versus the $2 \mathrm{~W}$ group; ${ }^{\&} p<0.05$ versus the $4 \mathrm{~W}$ group. 
Furthermore, there was a significant difference between the mRNA levels at 4 versus 2 weeks $(p<0.05)$. There was also a significant difference between the mRNA levels in the anterior and posterior joint capsules of rabbits immobilized for 6 versus 4 weeks $(p<0.05)$. The mRNA levels of TGF- $\beta 1$ in the anterior and posterior joint capsules of rabbits immobilized for 8 weeks tended to be increased compared with the values in rabbits immobilized for 6 weeks, but this difference failed to reach statistical significance $(p>0.05)$.

\section{Discussion}

Joint contracture is a pervasive complication of many orthopaedic or neurologic diseases. Once joint contracture is established, functional disturbances such as restricted joint activities, decreased strength of the muscles around the joint, and abnormal gait may be caused. ${ }^{2,12,25}$ Hence, quality of life in patients with the disease can be seriously affected. In clinical practice, joint immobilization is usually performed after the occurrence of a limb fracture or damage to other periarticular tissues to promote healing of the fracture and other joint tissues, decrease pain, and avoid further joint damage. ${ }^{16}$ However, joint contracture may also result from a long immobilization time and a fixed position of a single limb joint.

Animal models are established to study the processes that cannot be studied in humans, and to examine the effects of experimental interventions., ${ }^{2,26}$ An ideal animal model is of prime importance to the study of pathophysiological mechanism of a disease, and to the investigation of treatment selection and optimization. Up till the present moment, many researchers investigated joint contracture with animal model of flexion joint contracture. Chimoto et $\mathrm{al}^{27}$ immobilized the knee joints of rats with an internal fixator with the knee joint flexed at 150 degrees to develop animal model of flexion joint contracture and investigated the progression of an arthrogenic motion restriction after immobilization. The study indicated that joint contracture progressed rapidly until 8 weeks and then advanced slowly. Sasabe et $\mathrm{al}^{6}$ immobilized the knee joints of rats in full flexion using plaster casts to create animal model of flexion joint contracture and then examined the timedependent changes in the development of joint capsule fibrosis and in the number of myofibroblasts in the joint capsule after immobilization. The results suggested that joint capsule fibrosis with overexpression of type I collagen occurred and progressed within 1 week after immobilization, and an increase in myofibroblasts was related to the mechanism of joint capsule fibrosis. Nevertheless, extending joint contracture, which usually occurs in the knee joint, is the most common contracture type in clinic. To be close to clinical practice, we established an animal model of extending knee joint contracture with plaster cast in our study. The clinical characterization of joint contracture is the loss of joint ROM. The present study investigated joint contracture secondary to immobility longitudinally over 8 weeks using standard experimental conditions and quantitative tools. The present results demonstrated that rabbits that underwent joint immobilization experienced loss of joint ROM. A decrease in ROM was measurable after only 2 weeks of immobilization, and this constantly progressed for the first 8 weeks. Furthermore, our study indicated that the loss of ROM progressed rapidly in the first 6 weeks after immobilization, and then may progress slowly.

The formation of joint contracture results in stiffness of the capsule, synovial atrophy, fibrosis, and adhesion, which may be the causes of the limited ROM. ${ }^{12,28,29}$ In our study, synovial thickening of both the anterior and posterior joint capsules was found as early as 2 weeks after joint immobilization, and progressed throughout the 8-week study period. The thickening of the joint capsule progressed quickly in the first 6 weeks, and then seemed to progress more slowly. These results were consistent with the measurements of joint ROM, which indicated that the synovial thickening of the suprapatellar and posterior joint capsules may be related to the limited joint ROM. Furthermore, our study indicated that B ultrasonic examination can be a useful method for measuring the severity of joint contracture.

After the formation of joint contracture, histologic preparations revealed increased collagen synthesis and deposition in the contracted joint capsule. ${ }^{23}$ Hildebrand et $\mathrm{al}^{22}$ found that the mRNA and protein levels of collagen types I and III were both elevated in the experimental capsules compared with the control capsules in a rabbit knee contracture model. In our study, the collagen deposition in both the anterior and posterior joint capsules increased throughout the 8-week study period. Furthermore, the collagen deposition progressed quickly in the first 6 weeks, and then appeared to progress more slowly. The results were consistent with the measurements of joint ROM, which indicated that the collagen deposition in the anterior and posterior joint capsules may be related to the limited joint ROM to some extent.

TGF- $\beta 1$ is regarded as an important cytokine in the process of joint contracture, and is related to joint capsule fibrosis. ${ }^{23,30}$ Previous research investigating immobilized normal rabbit knees has shown alterations in TGF- $\beta 1$ expression. ${ }^{22,23}$ In our study, the mRNA expression of TGF- $\beta 1$ changed very early within the joint capsule in this rabbit model of joint contracture. The mRNA levels of TGF- $\beta 1$ in the anterior and posterior joint capsules altered within 2 weeks after joint immobilization, and progressed throughout the 8 -week study period. The mRNA expression levels increased quickly in the first 6 weeks, and then appeared to progress slowly, which coincided with the other measured variables. The increase in the mRNA levels of TGF- $\beta 1$ may be related to the collagen deposition in the anterior and posterior joint capsules. Joint capsule fibrosis may be concerned with the occurrence of joint contracture.

There are some limitations in our study. First, we only measured the mRNA levels of TGF- $\beta 1$; further work is required to evaluate the protein levels of TGF- $\beta 1$, and the mRNA and protein levels of other molecules and enzymes, to explore the possible mechanisms of the progression of joint contracture. Second, the apparatus used for ROM measurements in our study has some deficiencies. When the measurements were conducted, the force was not directly applied to the tibia but applied to the disc and then the tibia was indirectly turned. The force applied in the device was not always perpendicular to the tibia when the measurements were done, and the angle between the direction of force applied and the tibia changed 
as joint ROM changed. We will try to improve the methodology in our following studies. Furthermore, we only investigated the progression of joint contracture and the subsequent changes in the joint capsule; the changes in other periarticular tissues such as muscles and ligaments should be studied. Finally, we only investigated the progression of joint contracture for 8 weeks; thus, the longer term changes in periarticular tissues after joint immobilization are still uncertain. In our next work, we will investigate joint contracture after joint immobilization for a longer time period.

\section{Conclusion}

Our results indicated that rabbit knee joint immobilization can result in joint contracture; the severity of joint contracture progressed quickly in the first 6 weeks, and then may progress more slowly. The treatments evaluated in our study in this particular animal model might be considered to be geared toward the period of joint immobilization. Next, we should investigate the reversibility of joint contracture and explore the proper methods to prevent and treat joint contracture.

\section{Conflict of Interest}

None declared.

\section{References}

1 Kanazawa K, Hagiwara Y, Tsuchiya M, et al. Preventing effects of joint contracture by high molecular weight hyaluronan injections in a rat immobilized knee model. Int J Clin Exp Pathol 2015;8(04):3426-3440

2 Zhou Y, Zhang QB, Zhong HZ, Liu Y, Jing JH, Wu JX. Research progress on animal model of joint contracture. Austin Surg Case Rep 2016;1(02):1006

3 Wong K, Trudel G, Laneuville O. Intra-articular collagenase injection increases range of motion in a rat knee flexion contracture model. Drug Des Devel Ther 2017;12:15-24

4 Prabhu RK, Swaminathan N, Harvey LA. Passive movements for the treatment and prevention of contractures. Cochrane Database Syst Rev 2013;12(12):CD009331

5 Yoshinaga R, Ono T, Oki S, et al. The influence of 12-hour daily joint immobilization on the development of joint contracture in rats. Rigakuryoho Kagaku 2011;26(02):283-286

6 Sasabe R, Sakamoto J, Goto K, et al. Effects of joint immobilization on changes in myofibroblasts and collagen in the rat knee contracture model. J Orthop Res 2017;35(09):1998-2006

7 Kaneguchi A, Ozawa J, Kawamata S, Yamaoka K. Development of arthrogenic joint contracture as a result of pathological changes in remobilized rat knees. J Orthop Res 2017;35(07):1414-1423

8 Zhang QB, Zhou Y, Zhong HZ, Liu Y. Pathogenesis and progressing treatment of joint contracture. Zhonghua Chuangshang Guke Zazhi 2017;19(06):548-552

9 Dunham CL, Castile RM, Havlioglu N, Chamberlain AM, Galatz LM, Lake SP. Persistent motion loss after free joint mobilization in a rat model of post-traumatic elbow contracture. J Shoulder Elbow Surg 2017;26(04):611-618

10 Gruber G, Zacherl M, Giessauf C, et al. Quality of life after volar plate fixation of articular fractures of the distal part of the radius. J Bone Joint Surg Am 2010;92(05):1170-1178

11 Zhou Y, Wang F, Zhang QB, et al. Observation on curative effect of MOTOmed function training on patients with traumatic knee joint contracture. Zhonghua Linchuang Yishi Zazhi 2016;10(24): 3711-3715
12 Hagiwara Y, Saijo Y, Chimoto E, et al. Increased elasticity of capsule after immobilization in a rat knee experimental model assessed by scanning acoustic microscopy. Ups J Med Sci 2006;111(03):303-313

13 Moriyama H, Yoshimura O, Kawamata S, Takemoto H, Saka Y, Tobimatsu Y. Alteration of knee joint connective tissues during contracture formation in spastic rats after an experimentally induced spinal cord injury. Connect Tissue Res 2007;48(04):180-187

14 Lee S, Sakurai T, Ohsako M, Saura R, Hatta H, Atomi Y. Tissue stiffness induced by prolonged immobilization of the rat knee joint and relevance of AGEs (pentosidine). Connect Tissue Res 2010;51(06):467-477

15 Ando A, Hagiwara Y, Onoda Y, et al. Distribution of type A and B synoviocytes in the adhesive and shortened synovial membrane during immobilization of the knee joint in rats. Tohoku J Exp Med 2010;221(02):161-168

16 Trudel G, Seki M, Uhthoff HK. Synovial adhesions are more important than pannus proliferation in the pathogenesis of knee joint contracture after immobilization: an experimental investigation in the rat. J Rheumatol 2000;27(02):351-357

17 Monument MJ, Hart DA, Befus AD, Salo PT, Zhang M, Hildebrand KA. The mast cell stabilizer ketotifen fumarate lessens contracture severity and myofibroblast hyperplasia: a study of a rabbit model of posttraumatic joint contractures. J Bone Joint Surg Am 2010;92 (06):1468-1477

18 Sun Y, Li F, Fan C. Effect of pERK2 on extracellular matrix turnover of the fibrotic joint capsule in a post-traumatic joint contracture model. Exp Ther Med 2016;11(02):547-552

19 Clavet H, Hébert PC, Fergusson D, Doucette S, Trudel G. Joint contracture following prolonged stay in the intensive care unit. CMAJ 2008;178(06):691-697

20 Rodeo SA, Hannafin JA, Tom J, Warren RF, Wickiewicz TL. Immunolocalization of cytokines and their receptors in adhesive capsulitis of the shoulder. J Orthop Res 1997;15(03):427-436

21 Bunker TD, Reilly J, Baird KS, Hamblen DL. Expression of growth factors, cytokines and matrix metalloproteinases in frozen shoulder. J Bone Joint Surg Br 2000;82(05):768-773

22 Hildebrand KA, Zhang M, Germscheid NM, Wang C, Hart DA. Cellular, matrix, and growth factor components of the joint capsule are modified early in the process of posttraumatic contracture formation in a rabbit model. Acta Orthop 2008;79(01): $116-125$

23 Monument MJ, Hart DA, Befus AD, Salo PT, Zhang M, Hildebrand KA. The mast cell stabilizer ketotifen reduces joint capsule fibrosis in a rabbit model of post-traumatic joint contractures. Inflamm Res 2012r;61(04):285-292

24 Hildebrand KA, Zhang M, Hart DA. Joint capsule matrix turnover in a rabbit model of chronic joint contractures: correlation with human contractures. J Orthop Res 2006;24(05):1036-1043

25 Zhou Y, Yang X, Zhang QB, et al. Clinical effects of physical agents combined with exercise therapy on traumatic knee joint contracture. Shengwu Yixue Gongcheng Yu Linchuang 2017;21(03):289-293

26 Hildebrand KA, Sutherland C, Zhang M. Rabbit knee model of post-traumatic joint contractures: the long-term natural history of motion loss and myofibroblasts. J Orthop Res 2004;22(02): 313-320

27 Chimoto E, Hagiwara Y, Ando A, Itoi E. Progression of an arthrogenic motion restriction after immobilization in a rat experimental knee model. Ups J Med Sci 2007;112(03):347-355

28 Hagiwara Y, Ando A, Onoda Y, et al. Expression patterns of collagen types I and III in the capsule of a rat knee contracture model. J Orthop Res 2010r;28(03):315-321

29 Trudel G, Uhthoff HK. Contractures secondary to immobility: is the restriction articular or muscular? An experimental longitudinal study in the rat knee. Arch Phys Med Rehabil 2000;81(01):6-13

30 Hagiwara Y, Chimoto E, Takahashi I, Ando A, Sasano Y, Itoi E. Expression of transforming growth factor-beta1 and connective tissue growth factor in the capsule in a rat immobilized knee model. Ups J Med Sci 2008;113(02):221-234 